\title{
Sequential Resource Distribution Technique for Multi-User OFDM-SWIPT based Cooperative Networks
}

\author{
Sumit Gautam, Eva Lagunas, Symeon Chatzinotas and Björn Ottersten \\ Interdisciplinary Centre for Security, Reliability and Trust (SnT) \\ University of Luxembourg, 29, avenue JF Kennedy, L-1855 Luxembourg \\ Email: \{sumit.gautam,eva.lagunas,symeon.chatzinotas,bjorn.ottersten\}@uni.lu
}

\begin{abstract}
In this paper, we investigate resource allocation and relay selection in a dual-hop orthogonal frequency division multiplexing (OFDM)-based multi-user network where amplifyand-forward (AF) enabled relays facilitate simultaneous wireless information and power transfer (SWIPT) to the end-users. In this context, we address an optimization problem to maximize the end-users' sum-rate subjected to transmit power and harvested energy constraints. Furthermore, the problem is formulated for both time-switching (TS) and power-splitting (PS) SWIPT schemes. We aim at optimizing the users' SWIPT splitting factors as well as sub-carrier-destination assignment, sub-carrier pairing, and relay-destination coupling metrics. This kind of joint evaluation is combinatorial in nature with non-linear structure involving mixed-integer programming. In this vein, we propose a sub-optimal low complex sequential resource distribution (SRD) method to solve the aforementioned problem. The performance of the proposed SRD technique is compared with a semi-random resource allocation and relay selection approach. Simulation results reveal the benefits of the proposed design under several parameter values with various operating conditions to illustrate the efficiency of SWIPT schemes for the proposed techniques.
\end{abstract}

\section{INTRODUCTION}

The future of wireless communication enabled devices is full of many challenges, precisely in terms of capacity and performance demands. Additionally, the growing volumes of data traffic calls for energy-efficient network-layouts mainly due to energy management related issues. On the other hand, ensuring satisfactory services become even more challenging when the end-users are located at large distances from the transmit source. In this vein, cooperative relaying has emerged as useful means to assist a transmitter to communicate with the intended receiver by guaranteeing an improved coverage and appreciable overall throughput [1], [2].

Several devices within a network can assist the transmit node in relaying the relevant information to a far placed receiving node and vice-versa. Such devices, termed as relays, may adopt various cooperative strategies like regenerative (e.g., decode-and-forward (DF) [3]), and nonregenerative (e.g., amplify-and-forward (AF) [4]). In practice, non-regenerative relaying strategy is easy to implement with freedom to choose different transmit modulation schemes [5].

Simultaneous Wireless Information and Power Transfer (SWIPT) has recently been introduced as a promising technology for enabling the receiver to not only decode information from the received signal, but also harvest energy from the same electromagnetic (EM) signal, while ensuring adequate quality of service (QoS) [6], [7]. Two widely adopted SWIPTenabled receiver architectures are time-switching (TS), where energy is harvested from the received signal for a first few symbol durations in a block, while the data processing is performed for the remaining time duration; and power splitting (PS), where a fraction of received signal power is used for harvesting energy while the rest is sent to the information decoder.

One of the widely adopted spectral efficient multi-carrier transmission techniques, known as orthogonal frequency division multiplexing (OFDM), has been used with many recent standards and technologies including cooperative networks [8], [9], and SWIPT [10], [11]. When incorporated with wireless energy transfer, OFDM not only preserves its existing advantages, but it also allows multiple devices to harvest energy simultaneously using the same received EM signal.

Numerous works have been carried out in the literature which considers OFDM resource allocation both in conventional and relay-aided communication networks [12], [13]. Techniques to flexibly assign the OFDM sub-carriers based on channel state information (CSI) have been presented in [14], [15], where joint sub-carrier and power allocation is performed in relay-aided systems. However, the abovementioned works neither consider energy harvesting, nor do they consider relay selection together with transmitted power and sub-carrier assignment. In [16], the author presented a framework to study SWIPT at the relays in a two-hop multirelay-assisted single user system, where relay selection and OFDM are not taken into consideration. Interestingly, incorporation of relaying technologies with multi-user multicarrier systems can offer significant gains in terms of channel diversity for different users, particularly when the sourcedestination pairs are placed far apart. Moreover, cooperative communication can provide additional advantages to improve the SWIPT performance. In particular, the power transfer distance is largely limited by the power sensitivity of the energy harvester, which considering the current state-of-theart technology is of $-10 \mathrm{dBm}$, significantly tighter than the $-60 \mathrm{dBm}$ assumed for effective information receivers [17]. The benefits of cooperative transmission for OFDM-SWIPT 


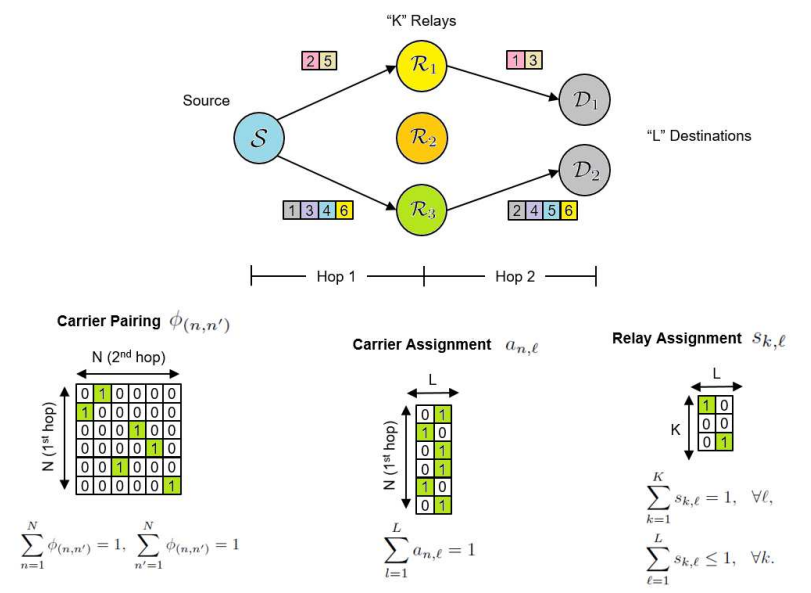

Fig. 1: Multi-relay multi-user OFDM-based system model with $K=3$ and $L=2$.

are much less investigated [18], [19]. Recently, relay selection along with the design of the optimal SWIPT splitting factors has been addressed in [20], [21] for a two-hop relaying network considering power harvesting constraints at the receiver.

Unlike the previous works, in this paper, we consider single source assisted by multiple half-duplex AF-enabled relays based on OFDM to forward both information and energy to multiple users. Considering this two-hop relay-assisted multi-user network with SWIPT, we investigate single relay selection from a pool of available relays and optimize the subcarrier pairing, power allocation, and SWIPT splitting factors. In order to significantly reduce the control and synchronization process among the relay nodes, single relay is chosen over multiple relays. We formulate a problem to optimize the relay selection and resource allocation based on maximization of the end-users' sum-rate constrained to minimum individual user's harvested energy and transmit power limits. Due to joint optimization of several resources, an analysis within the full search space imposes very high complexities thereby making the problem extremely challenging to solve. To circumvent this tedious and unaffordable optimization, we seek for a less complex heuristic solution. In this context, we propose a novel sequential resource distribution (SRD) algorithm based on pairing the maximum channel gains of both the hops to maximize the end-users' sum-rate by jointly performing optimization of sub-carrier-destination assignment, sub-carrier pairing, and relay-destination coupling metrics, followed by power allocation to sub-carriers in each hop, and computation of SWIPT splitting factors at the destination.

The remainder of this paper is organized as follows. Section II introduces the system model. The problem formulation and the proposed solution are presented in Section III and Section IV, respectively. Brief analysis to distinguish between the computational complexities of proposed methods is discussed in Section V. Numerical results are shown in Section VI, followed by concluding remarks in Section VII.

\section{System Model}

The system model considered in this paper consists of single source $\mathcal{S}$, that transfers information and power to $L$

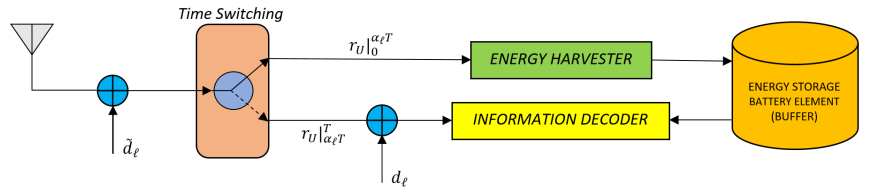

Fig. 2: User receiver architecture based on TS scheme.

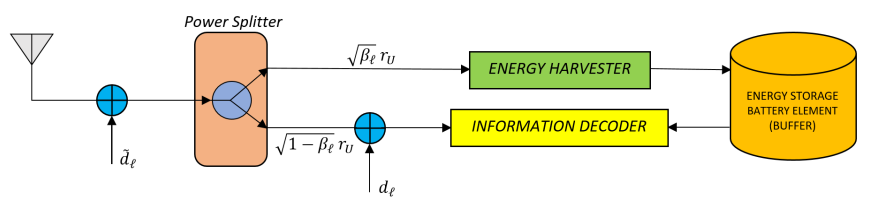

Fig. 3: User receiver architecture based on PS scheme.

destinations $\left(\mathcal{D}_{1}, \ldots, \mathcal{D}_{L}\right)$ via a set of $K$ non-regenerative half-duplex relays $\left(\mathcal{R}_{1}, \ldots, \mathcal{R}_{K}\right)$, where $L \leq K$. The source, relay and destination nodes are equipped with single antenna. The direct source-destination link is discarded as it is assumed to suffer strong attenuation. Two orthogonal time slots accounts for the overall communication process. The source transmits the signal to the selected set of relay nodes in the first time slot while the chosen relays forward an amplified version of the received signal to the intended destinations over the second time slot. Further, a relay is assigned to one destination only, and is not to be shared with other nodes. We consider an OFDM-based transmission with $N$ orthogonal flat-fading sub-carriers such that $1 \leq n \leq N$ and $1 \leq n^{\prime} \leq N$ denote the sub-carriers in the first and second hops, respectively. An exclusive sub-carrier pair $\left(n, n^{\prime}\right)$ is formed at each AF relay to assist successive transmissions in both the hops on a sub-carrier pair basis. The sub-carrier indices $n$ and $n^{\prime}$ may be equal or not. In addition, we assume that the assignment of each sub-carrier pair is restricted to one relay only, and each relay can acquire multiple unshared sub-carrier pairs. An example of relay selection and OFDM sub-carrier pairing for a multi-relay multi-user network with $N=6, K=3$ and $L=2$ is illustrated in Fig. 1. Details on the sub-carrier-destination assignment, sub-carrier pairing, and relay-destination coupling metrics will be discussed in the forthcoming sections.

Let us denote $h_{1, n, k}$ and $h_{2, n^{\prime}, k, \ell}$ as the first hop channel coefficient on the $n$-th sub-carrier between $\mathcal{S}$ and $\mathcal{R}_{k}$, and the second hop channel coefficient on the $n^{\prime}$-th sub-carrier between the $\mathcal{R}_{k}$ and $\mathcal{D}_{\ell}$, respectively. Denote $p_{1, n}$ as the first hop transmit power on the $n$-th sub-carrier at the source $\mathcal{S}$, while the following amplification coefficient is used by $\mathcal{R}_{k}$ to re-transmit the received signal

$$
g_{\left(n, n^{\prime}\right), k}=\sqrt{\frac{p_{2, n^{\prime}, k}}{p_{1, n}\left|h_{1, n, k}\right|^{2}+\sigma_{k}^{2}}},
$$

which ensures that the $k$-th relay transmits with a power $p_{2, n^{\prime}, k}$ on the $n^{\prime}$-th sub-carrier. In (1), $\sigma_{k}^{2}$ denotes the noise power at the $\mathcal{R}_{k}$ relay. The total available power at the source $\mathcal{S}$ and at the relay $\mathcal{R}_{k}$ is fixed as $P_{S}$ and $P_{\mathcal{R}, k}$, respectively.

We consider two distinct SWIPT designs at the destination $\mathcal{D}_{\ell}$ namely, TS and PS schemes. The receiver architecture for TS scheme is shown in Fig. 2. For a block transmission of duration $T$ seconds with $T=\hat{N} T_{s}\left(\hat{N}\right.$ and $T_{s}$ corresponding 
to the number of transmitted symbols per block and the symbol period, respectively), we define a time-switching ratio at the $\ell$-th user as $\alpha_{\ell}$, where $0 \leq \alpha_{\ell} \leq 1$ and $\ell=1,2, \ldots, L$, such that the energy is harvested from the received signal for the first $\alpha_{\ell} \hat{N} T_{s}$ second while data processing takes place for the remaining duration.

In case of the PS receiver as depicted in Fig. 3, a power splitter is employed so that a fraction $\sqrt{\beta_{\ell}}$, where $0 \leq \beta_{\ell} \leq 1$, of the received signal power is used for energy harvesting, while the remaining is sent to the information decoder.

Furthermore, we normalize the transmission time in each hop to use energy and power terms interchangeably. The practical antenna noise, $\tilde{d}_{\ell} \in \mathcal{C N}\left(0, \sigma_{\tilde{d}_{\ell}}^{2}\right)$, has a negligible impact on both the data processing and energy harvesting due to extremely lower values of $\sigma_{\tilde{d}_{\ell}}^{2}$ in comparison to the baseband processing circuit noise, and the average power of the received signal [22]. Therefore, we ignore the noise term $\tilde{d}_{\ell}$ in the following analysis. The relay nodes are assumed to have their own power supply and thus, do not harvest energy.

The effective signal-to-noise ratio (SNR) seen at the decoding branch of the destination $\mathcal{D}_{\ell}$ for the $\left(n, n^{\prime}\right)$ sub-carrier pair over the $\mathcal{S} \rightarrow \mathcal{R}_{k} \rightarrow \mathcal{D}_{\ell}$ link corresponding to the TS and PS scheme is respectively given by,

$$
\begin{array}{r}
\gamma_{\left(n, n^{\prime}\right), k, \ell}^{T S}=\frac{p_{1, n}\left|h_{1, n, k} g_{\left(n, n^{\prime}\right), k} h_{2, n^{\prime}, k, \ell}\right|^{2}}{\sigma_{k}^{2}\left|h_{2, n^{\prime}, k, \ell} g_{\left(n, n^{\prime}\right), k}\right|^{2}+\sigma_{d_{\ell}}^{2}}, \\
\gamma_{\left(n, n^{\prime}\right), k, \ell}^{P S}=\frac{\left(1-\beta_{\ell}\right) p_{1, n}\left|h_{1, n, k} g_{\left(n, n^{\prime}\right), k} h_{2, n^{\prime}, k, \ell}\right|^{2}}{\left(1-\beta_{\ell}\right) \sigma_{k}^{2}\left|h_{2, n^{\prime}, k, \ell} g_{\left(n, n^{\prime}\right), k}\right|^{2}+\sigma_{d_{\ell}}^{2}},
\end{array}
$$

where $\sigma_{d_{\ell}}^{2}$ denotes the noise power introduced by the downconversion procedure at $\mathcal{D}_{\ell}$. The above expressions can be simplified and re-written as follows

$$
\begin{array}{r}
\gamma_{\left(n, n^{\prime}\right), k, \ell}^{T S}=\frac{\gamma_{1, n, k} \gamma_{2, n^{\prime}, k, \ell}}{1+\gamma_{1, n, k}+\gamma_{2, n^{\prime}, k, \ell}}, \\
\gamma_{\left(n, n^{\prime}\right), k, \ell}^{P S}=\frac{\left(1-\beta_{\ell}\right) \gamma_{1, n, k} \gamma_{2, n^{\prime}, k, \ell}}{1+\gamma_{1, n, k}+\left(1-\beta_{\ell}\right) \gamma_{2, n^{\prime}, k, \ell}},
\end{array}
$$

where $\gamma_{1, n, k}=\frac{p_{1, n}\left|h_{1, n, k}\right|^{2}}{\sigma_{k}^{2}}$, and $\gamma_{2, n^{\prime}, k, \ell}=\frac{p_{2, n^{\prime}, k}\left|h_{2, n^{\prime}, k, \ell}\right|^{2}}{\sigma_{d_{\ell}}^{2}}$. Assuming that the SNR of the relayed signal is high, we can further simplify the expressions by applying the following approximation,

$$
\begin{array}{r}
\hat{\gamma}_{\left(n, n^{\prime}\right), k, \ell}^{T S} \approx \frac{\gamma_{1, n, k} \gamma_{2, n^{\prime}, k, \ell}}{\gamma_{1, n, k}+\gamma_{2, n^{\prime}, k, \ell}}, \\
\hat{\gamma}_{\left(n, n^{\prime}\right), k, \ell}^{P S} \approx \frac{\left(1-\beta_{\ell}\right) \gamma_{1, n, k} \gamma_{2, n^{\prime}, k, \ell}}{\gamma_{1, n, k}+\left(1-\beta_{\ell}\right) \gamma_{2, n^{\prime}, k, \ell}} .
\end{array}
$$

The aforementioned approximation is commonly used in the literature to make the problem more tractable [14], [23].

The throughput achieved by the corresponding TS and PS decoding branch of the $\left(n, n^{\prime}\right)$ sub-carrier pair over the $\mathcal{S} \rightarrow$ $\mathcal{R}_{k} \rightarrow \mathcal{D}_{\ell}$ link can thus be expressed as

$$
\begin{array}{r}
R_{\left(n, n^{\prime}\right), k, \ell}^{T S}=\frac{1}{2}\left(1-\alpha_{\ell}\right) \ln \left(1+\hat{\gamma}_{\left(n, n^{\prime}\right), k, \ell}^{T S}\right), \\
R_{\left(n, n^{\prime}\right), k, \ell}^{P S}=\frac{1}{2} \ln \left(1+\hat{\gamma}_{\left(n, n^{\prime}\right), k, \ell}^{P S}\right),
\end{array}
$$

where the factor $1 / 2$ is introduced to compensate for the two time slots of the considered relay assisted communication.
On the other hand, the energy harvested at the harvesting branch for the $\left(n, n^{\prime}\right)$ sub-carrier pair over the $\mathcal{S} \rightarrow \mathcal{R}_{k} \rightarrow \mathcal{D}_{\ell}$ link corresponding to the TS and PS schemes is given by

$$
\begin{aligned}
& E_{\left(n, n^{\prime}\right), k, \ell}^{T S}=\zeta \alpha_{\ell}\left[\left|g_{\left(n, n^{\prime}\right), k} h_{2, n^{\prime}, k, \ell}\right|^{2}\left(p_{1, n}\left|h_{1, n, k}\right|^{2}+\sigma_{k}^{2}\right)\right], \\
& E_{\left(n, n^{\prime}\right), k, \ell}^{P S}=\zeta \beta_{\ell}\left[\left|g_{\left(n, n^{\prime}\right), k} h_{2, n^{\prime}, k, \ell}\right|^{2}\left(p_{1, n}\left|h_{1, n, k}\right|^{2}+\sigma_{k}^{2}\right)\right]
\end{aligned}
$$

which can be simplified by using the expression in (1), resulting in,

$$
\begin{aligned}
& E_{\left(n, n^{\prime}\right), k, \ell}^{T S}=\zeta \cdot \alpha_{\ell} \cdot p_{2, n^{\prime}, k}\left|h_{2, n^{\prime}, k, \ell}\right|^{2}, \\
& E_{\left(n, n^{\prime}\right), k, \ell}^{P S}=\zeta \cdot \beta_{\ell} \cdot p_{2, n^{\prime}, k}\left|h_{2, n^{\prime}, k, \ell}\right|^{2},
\end{aligned}
$$

where $\zeta$ is the energy conversion efficiency of the receiver. The following triplet is defined to further simplify the notation

$$
\left(\hat{R}_{\left(n, n^{\prime}\right), k, \ell} ; \hat{E}_{\left(n, n^{\prime}\right), k, \ell} ; \theta_{\ell}\right)=\left\{\begin{array}{l}
\left(R_{\left(n, n^{\prime}\right), k, \ell}^{T S} ; E_{\left(n, n^{\prime}\right), k, \ell}^{T S} ; \alpha_{\ell}\right) \\
\left(R_{\left(n, n^{\prime}\right), k, \ell}^{P S} ; E_{\left(n, n^{\prime}\right), k, \ell}^{P S} ; \beta_{\ell}\right)
\end{array},\right.
$$

corresponding to the TS and PS schemes, respectively.

\section{Problem Formulation}

We aim at designing the sub-carrier pairing, relay selection, power allocation and SWIPT splitting factors' optimization so that the total end-to-end users' sum-rate is maximized subject to a set of transmitted power and harvested energy constraints.

Let us denote $\phi_{\left(n, n^{\prime}\right)} \in\{0,1\}$ as the indicator for subcarrier pairing, where $\phi_{\left(n, n^{\prime}\right)}=1$ means that sub-carrier $n$ in the first hop is paired with sub-carrier $n^{\prime}$ of the second hop and $\phi_{\left(n, n^{\prime}\right)}=0$ otherwise. Each sub-carrier in the first hop can be paired with one and only one sub-carrier of the second hop. The binary variable $\phi_{\left(n, n^{\prime}\right)}$ must satisfy,

$$
\sum_{n=1}^{N} \phi_{\left(n, n^{\prime}\right)}=1, \forall n^{\prime} ; \quad \sum_{n^{\prime}=1}^{N} \phi_{\left(n, n^{\prime}\right)}=1, \forall n .
$$

For the relay selection problem, we make use of the binary variable $s_{k, \ell}=\{0,1\}$, where $s_{k, \ell}=1$ means that $\mathcal{R}_{k}$ is selected for $\mathcal{D}_{\ell}$. It is clear that one relay will be allocated to one and only one user, and thus,

$$
\sum_{k=1}^{K} s_{k, \ell}=1, \forall \ell ; \quad \sum_{\ell=1}^{L} s_{k, \ell} \leq 1, \forall k .
$$

In order to link each sub-carrier pair with the corresponding destination node, we define $a_{n, \ell} \in\{0,1\}$ as the binary variable with $a_{n, \ell}=1$ indicating that sub-carrier $n$ is used in the first hop for reaching destination $\mathcal{D}_{\ell}$, and $a_{n, \ell}=0$ otherwise. Therefore, the following assignment rule must hold

$$
\sum_{\ell=1}^{L} a_{n, \ell}=1, \quad \forall n .
$$

The rate achieved at $\mathcal{D}_{\ell}$ can be formulated using the previous variable definitions with (14), represented as

$$
\hat{R}_{\ell}=\sum_{i=1}^{L} \sum_{k=1}^{K} \sum_{n=1}^{N} \sum_{n^{\prime}=1}^{N} a_{n, \ell} \phi_{\left(n, n^{\prime}\right)} s_{k, \ell} \hat{R}_{\left(n, n^{\prime}\right), k, i} .
$$

Regarding the power constraints, we assume

$$
\sum_{n=1}^{N} p_{1, n} \leq P_{S},
$$




$$
\sum_{n^{\prime}=1}^{N} \sum_{\ell=1}^{L} \sum_{n=1}^{N} s_{k, \ell} a_{n, \ell} \phi_{\left(n, n^{\prime}\right)} p_{2, n^{\prime}, k} \leq P_{R, k}, \quad k=1, \cdots, K,
$$

where (C4) and (C5) represents the source power constraint and the relay power constraints, respectively.

On the other hand, we consider all the intended sub-carriers to represent the overall energy harvested at $\mathcal{D}_{\ell}$ as follows

$$
\hat{E}_{\ell}=\sum_{k=1}^{K} \sum_{i=1}^{L} \sum_{n=1}^{N} \sum_{n^{\prime}=1}^{N} s_{k, \ell} \phi_{\left(n, n^{\prime}\right)} \hat{E}_{\left(n, n^{\prime}\right), k, i} .
$$

As a consequence, the minimum harvested power constraint at destination $\mathcal{D}_{\ell}$ can be stated as follows,

$$
\hat{E}_{\ell} \geq \eta_{\ell}, \quad \ell=1, \cdots, L,
$$

where $\eta_{\ell}$ is the minimum harvested power demanded by $\mathcal{D}_{\ell}$.

Therefore, the proposed optimization problem can mathematically be represented as follows,

$$
\begin{aligned}
(P 1): \max _{\{\boldsymbol{\phi}, \mathbf{s}, \mathbf{a}, \mathbf{p}, \boldsymbol{\theta}\}} & \sum_{\ell=1}^{L} \hat{R}_{\ell} \\
\text { subject to : } & (C 1),(C 2),(C 3),(C 4),(C 5),(C 6), \\
& (C 7): 0 \leq \theta_{\ell} \leq 1, \ell=1, \cdots, L,
\end{aligned}
$$

where $\boldsymbol{\phi}=\left\{\phi_{\left(n, n^{\prime}\right)}\right\}, \mathbf{s}=\left\{s_{k, \ell}\right\}, \mathbf{a}=\left\{a_{n, \ell}\right\}, \mathbf{p}=$ $\left\{p_{1, n}, p_{2, n^{\prime}, k}\right\}$ and $\boldsymbol{\theta}=\left\{\theta_{\ell}\right\}$ denote the optimization variables corresponding to sub-carrier pairing, relay selection, carrierdestination assignment, power allocation, and the SWIPT splitting factors, respectively. Herein, we assume that an optimal solution exists for $(P 1)$ and therefore the problem is feasible. However, obtaining an optimal solution of this problem is challenging due to the lack of convexity introduced by joint optimization of resource allocation variables and presence of mixed-integer variables. Therefore, we propose a heuristic method to deal with the optimization problem in $(P 1)$. We assume that the source node has full CSI, and hence capable of solving this optimization problem. Subsequently, it forwards the instructions to the other nodes via proper signaling.

\section{Proposed Solution Based on SEquential RESOURCE Distribution (SRD) TECHNiQUE}

The proposed solution is divided into three steps: First, the relay selection, sub-carrier pairing and carrier-destination variables $(\phi, \mathbf{s}, \mathbf{a})$ are jointly determined based on the proposed sequential resource distribution (SRD) algorithm. Next, the transmitted powers (p) are optimized based on conventional Water-Filling (WF) over the assigned links. Finally, the SWIPT splitting factors $(\boldsymbol{\theta})$ are computed in order to satisfy (C6). Each step is described in detail in the following sections.

\section{A. Relay selection and sub-carrier assignment}

In this section, we propose a sequential resource distribution (SRD) algorithm, inspired by the backtracking algorithm, to maximize the end-to-end performance of two-hop nonregenerative systems by pairing the maximum SNRs of the two hops, as depicted on the top of next column. Intuitively,

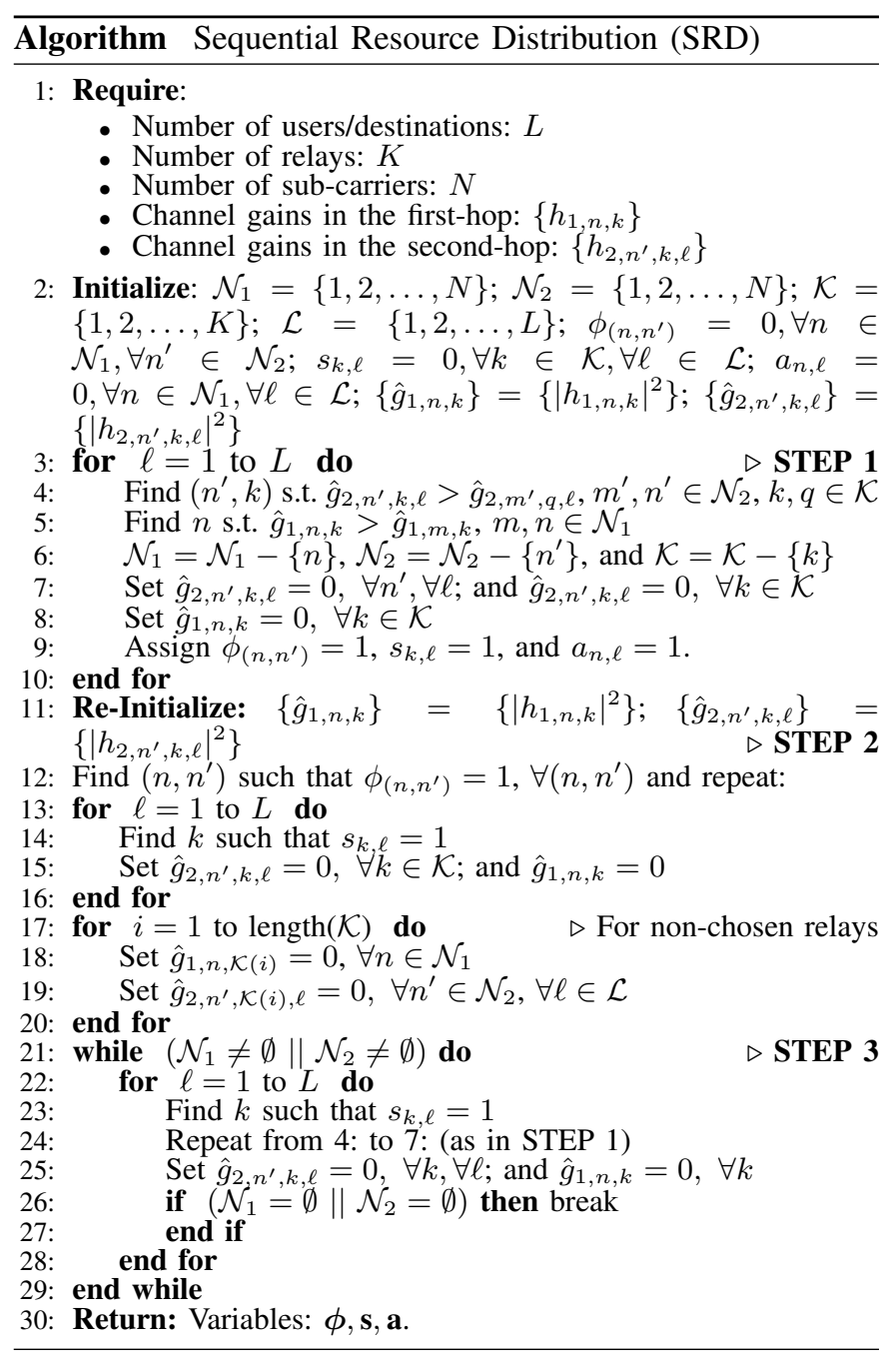

as there is no interference in the considered system, therefore maximizing harvested energy or information transfer translates into maximizing the SNR. To proceed, we first find the maximum channel gain coefficient in the second hop to fix the indices corresponding to the second hop subcarrier and the relay i.e., $n^{\prime}$ and $k$ in the first step of the algorithm. Ensuring strong relay-destination link motivates for the use of backtracking approach-inspired SDR method. Correspondingly, we also find the maximum channel gain coefficient in the first hop for the $k$-th relay chosen as above, and fix the maximum sub-carrier $n$ to form the sub-carrier pair $\left(n, n^{\prime}\right)$. We follow a mechanism which allows relay allocation to the destination based on first come first serve scheduling. For better insight, let us consider the set-up as in Fig. 1 and assume that $n^{\prime}=4$ and $k=2$ is the first assignment. Then $n^{\prime}=4$ cannot be allocated again to any other relay. Similarly, $k=2$ should not be paired with any other destination and hence all the remaining sub-carriers for this relay are set to inactive state. It should be noted that step 1 is sequentially repeated until all destinations are assigned and this fixes the relay-destination coupling matrix (s) and an incomplete version of the variables $\phi$ and $\mathbf{a}$, since 


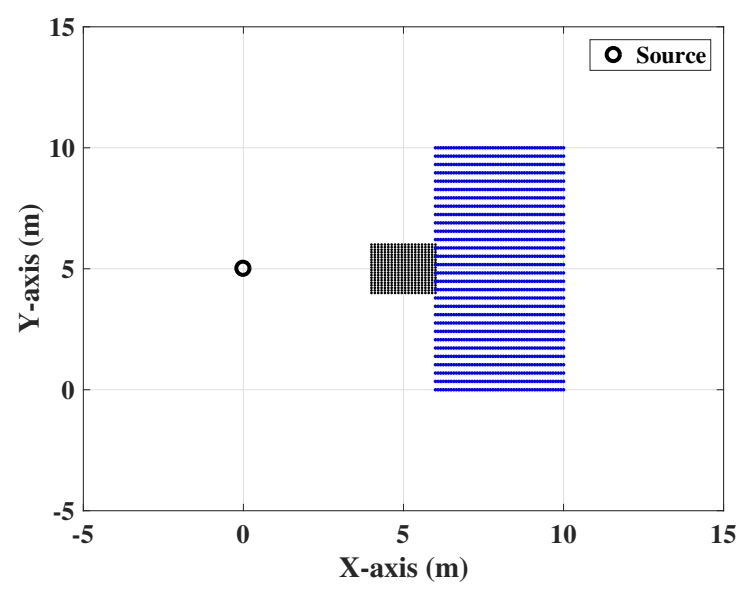

Fig. 4: Simulated scenario.

only the sub-carrier pairs associated with the assigned relays have been determined. In step 2, we reform both the channel matrices, which are composed using $\left\{h_{1, n, k}\right\}$ and $\left\{h_{2, n^{\prime}, k, \ell}\right\}$, corresponding to the two-hops where we remove all the values of relay indices for already selected $n$ and $n^{\prime}$. Additionally, we also discard the non-chosen relays by eliminating the values of corresponding relay indices. Finally in step 3 , we allocate the remaining resources by performing the sub-carrier assignment and relay selection as in step 1 , followed by removal of subcarrier indices for the chosen relay. Consequently, the SRD algorithm returns the complete version of the variables $\phi, \mathbf{s}$, and $\mathbf{a}$.

\section{B. Power allocation}

After having obtained suitable solutions of sub-carrierdestination assignment (s), sub-carrier pairing $(\phi)$, and relaydestination coupling (a) metrics, the transmitted power $\mathbf{p}=$ $\left\{p_{1, n}, p_{2, n^{\prime}, k}\right\}$ can be efficiently assigned according to the channel conditions by using the well-known WF strategy. In this context, we consider a WF approach for the source and for each of the active relay nodes. Therefore, the transmitted power on the first hop is given by,

$$
p_{1, n}= \begin{cases}\frac{1}{\nu}-\varphi_{1, n}, & \text { if } \nu<\frac{1}{\varphi_{1, n}} \\ 0, & \text { otherwise }\end{cases}
$$

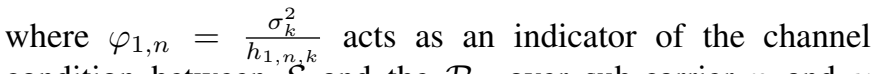
condition between $\mathcal{S}$ and the $\mathcal{R}_{k}$ over sub-carrier $n$ and $\nu$ is the decision threshold.

Similarly, the transmitted power on the second hop for the $k$-th relay is determined as,

$$
p_{2, n^{\prime}, k}= \begin{cases}\frac{1}{\nu}-\varphi_{2, n^{\prime}, k}, & \text { if } \nu<\frac{1}{\varphi_{2, n^{\prime}, k}} \\ 0, & \text { otherwise, }\end{cases}
$$

where $\varphi_{2, n^{\prime}, k}=\frac{\sigma_{\ell}^{2}}{h_{2, n^{\prime}, k, \ell}}$ acts as an indicator of the channel condition between $\mathcal{R}_{k}$ and $\mathcal{D}_{\ell}$ over sub-carrier $n^{\prime}$.

\section{Computation of the SWIPT splitting factors}

In this section, we address the computation of SWIPT splitting factors $\boldsymbol{\theta}=\left\{\theta_{\ell}\right\}$. The proposed solution is found by letting equality hold for the set of constraints in (C6). Therefore, the optimal values $\theta_{\ell}^{*}$ are given by,

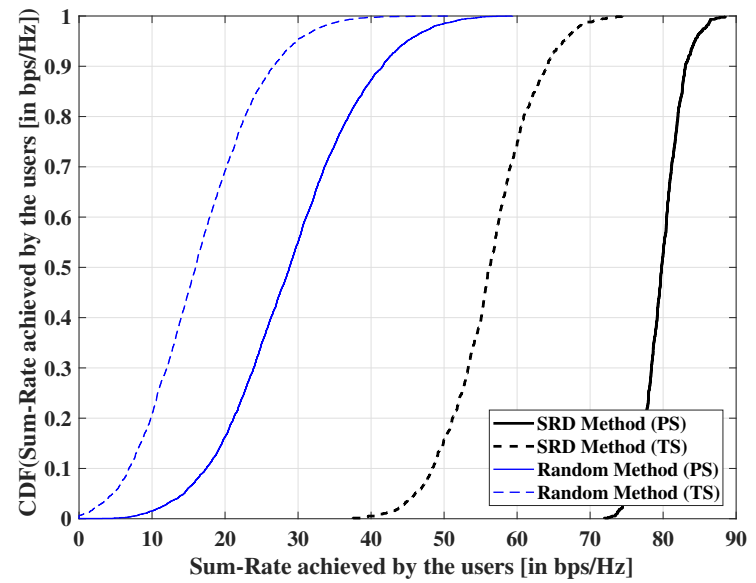

Fig. 5: CDF of sum-rate distribution: $K=3, L=2, \eta=1 \mu \mathrm{J}$.

$$
\theta_{\ell}^{*}=\frac{\eta_{\ell}}{E_{\ell, \max }},
$$

where $E_{\ell, \max }$ is computed with (16) assuming $\theta_{\ell}=1$.

\section{Computational Complexity Analysis}

In this section, we present a brief discussion on the computational complexities of the proposed SRD method in contrast to an exhaustive search and random selection method. It is noteworthy that the proposed SRD approach with WF has a far lesser time complexity of $\mathcal{O}\left(K \cdot L^{\psi+2} \cdot N^{2}\right)$, where $L^{\psi}$ are the number of iterations for the loop between 21: and 29: of the proposed algorithm, in comparison to an optimal exhaustive search approach with $(K \cdot L)^{N !}$ possible combinations of $\phi, \mathbf{s}$, and a. The exhaustive search method becomes inconvenient for higher values of $K, L$, and $N$, due to extremely high time-complexity. However, the time-complexity of the random selection method with WF is very fast and of $\mathcal{O}\left(N^{2}\right)$, but using this kind of hit-and-trial technique has the probability of getting the optimal choice as $1 /(K \cdot L)^{N !}$. Therefore, the random selection method is considered to provide a suboptimal solution unless the hit-and-trial method coincides exactly with optimal selection.

\section{Simulation Results}

In this section, we present some simulation results to evaluate the performance of the proposed SRD technique. The emulations are performed for different parameter values to analyze the efficiency of proposed design. For simplicity, we assume that the source and all the relays are subject to the same power constraint i.e., $P_{S}=P_{R, 1}=P_{R, 2}=\ldots=P_{R, K}$, the harvested energy demand at each user is assumed to be same i.e, $\eta_{1}=\eta_{2}=\ldots=\eta_{\ell}=\eta$, and $\zeta=0.8$ throughout this paper.

We consider the node distribution as shown in Fig. 4, where we assume that a source is fixed at $(0,5) \mathrm{m}$, the $K$ relay nodes are located randomly inside a $4 \mathrm{~m}^{2}$ region and specifically within the coordinates $(4,4) \mathrm{m}$ and $(6,6)$ $\mathrm{m}$ between the source and the destination area. The endusers are placed randomly as well within a $4 \times 10 \mathrm{~m}^{2}$ area between the coordinates $(6,0) \mathrm{m}$ and $(10,10) \mathrm{m}$. The ITU Radiocommunication Sector (ITU-R) P.1238 channel model 


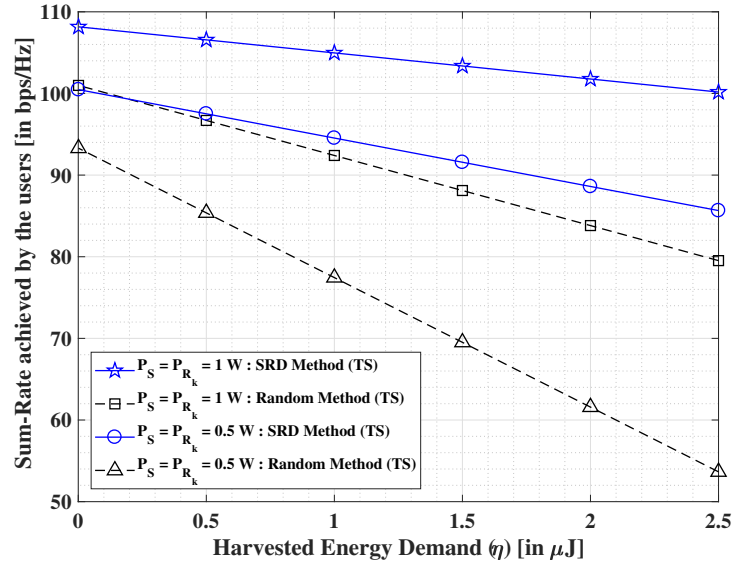

(a)

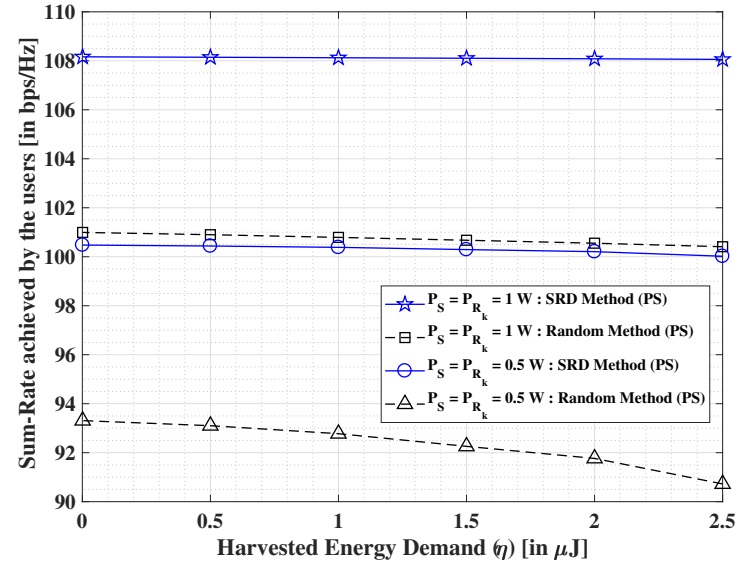

(b)

Fig. 6: Sum-rate achieved by all the users versus different harvested energy demands for various values of transmit powers with $K=6, L=4$, and $N=32$ to illustrate the performance of (a) TS scheme, and (b) PS scheme.

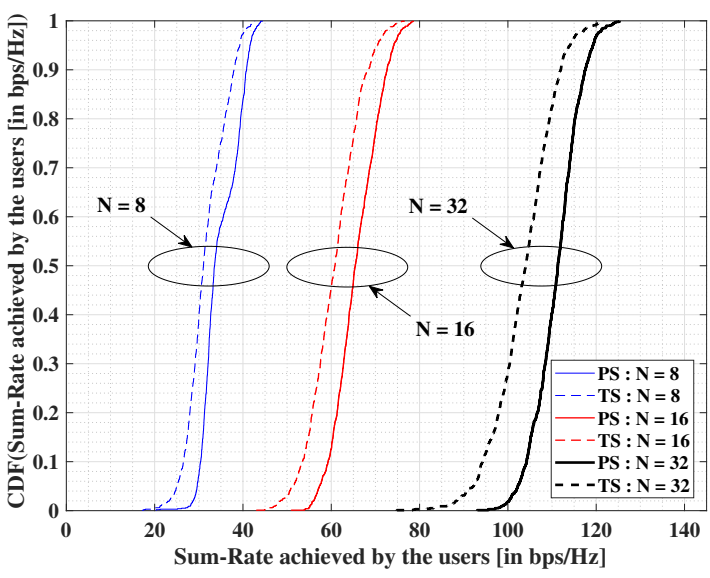

Fig. 7: CDF of sum-rate distribution: $K=5, L=3$.

is employed with the central frequency assumption at 1.9 $\mathrm{GHz}$ to emulate a wireless broadband network. We consider a frequency-selective channel model, with 5-multipath arrivals averaged according to the Poisson process and the root mean square (rms) delay is set to $36.3078 \mathrm{~ns}$ as per the aforementioned room dimensions. The signal fading from the source to the relays and from relays to the destinations follow the Ricean distribution with K-factor of 3.5. All the OFDM subcarriers are assumed to experience a flat-fading and the total bandwidth is fixed to $20 \mathrm{MHz}$. The effect from shadowing is considered negligible and is hence discarded in the system setup. The noise power at the relay and destination nodes is assumed the same i.e., $-110 \mathrm{dBW}$.

For comparison purposes, we consider a random relay and sub-carrier assignment, followed by the proposed power allocation and SWIPT splitting factors' assignment as in section IV-B and IV-C, respectively. This comparison method is chosen due to the lack of prior work in the specific problem and an unimplementable highly complex exhaustive search method. Fig. 5 compares the Cumulative Density Function (CDF) of the sum-rate achieved by the users after 750 Monte-Carlo random channel realizations of the proposed SRD and the random strategy in a network composed of

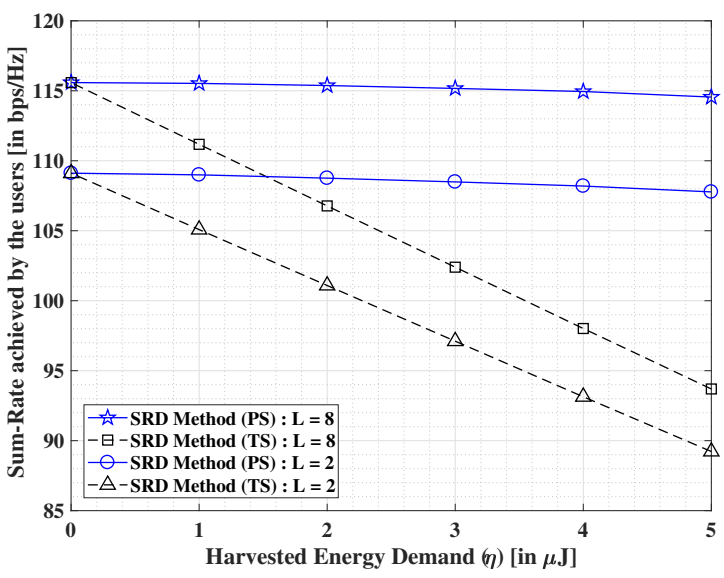

Fig. 8: Sum-rate versus demanded energy for various $L$.

$K=3$ relays and $L=2$ users, with $N=32$ OFDM sub-carriers and maximum transmitted power of $0.1 \mathrm{~W}$, i.e., $P_{S}=P_{R, 1}=P_{R, 2}=P_{R, 3}=0.1 \mathrm{~W}$. At each realization, different channel coefficients and different locations for the relay and destination nodes are selected. The demanded power $\eta$ is set to $1 \mu \mathrm{J}$ for all the destination nodes. We observe that the proposed SRD technique significantly outperforms the semi-random scheme with PS providing an additional performance advantage over TS for both the methods.

Next, we illustrate the phenomenon of rate-energy (RE) trade-off of the proposed SRD scheme in contrast to the random selection scheme considering both TS and PS architectures. Fig. 6(a) and Fig. 6(b) project the variation in the values of sum-rate for different harvested energy demand values with various transmit powers. We set $K=6, L=4$, $N=32$ and the emulation for each point in both the figures correspond to the mean of sum-rate obtained after 150 experiment realizations. Both Fig. 6(a) and Fig. 6(b) show the decreasing sum-rate with respect to the increasing harvested energy demands where the curve is linear in the case of TS, while it is non-linear in the case of PS. However, it is seen that the sum-rate increases appreciably with increasing transmit power values. As evident from the figures, the proposed SRD 
approach outperforms the random selection approach by a good margin. It is also noteworthy that the efficiency of the PS receiver architecture is proven to be considerably better than its TS counterpart.

To illustrate the gain provided by frequency diversity, Fig. 7 presents the CDF plot of the users' sum-rate for a fixed harvested energy demand of $\eta=0.75 \mu \mathrm{J}$ with $K=6$, $L=4$, and $P_{S}=P_{R, 1}=P_{R, 2}=\ldots=P_{R, 6}=0.75$ W for different values of $N$. The results are evaluated and averaged over 1000 Monte-Carlo random channel conditions. It is shown that the sum-rate keeps increasing with increasing values of $N$, thereby implying that the system performance improves as the number of sub-carriers increase. Additionally, it is observed that the PS receiver architecture outperforms the TS-based SWIPT receiver architecture.

Fig. 8 depicts the performance of the proposed SRD approach for different number of users with $K=10, N=32$, and $P_{S}=P_{R, 1}=P_{R, 2}=\ldots=P_{R, 10}=1.5 \mathrm{~W}$. Each point in the figure corresponds to the sum-rate mean achieved after 200 experiment realizations. However, as mentioned previously, it is seen that the sum-rate decreases with the increasing harvested energy demands. But interestingly, the sum-rate increases as the number of users increases provided there are sufficient network resources. Growing number of users invoke more relays thereby exploiting the network diversity which in-turn increases the overall system gain. Moreover, as noted in previous observations, it is worth mentioning that the performance of the PS architecture is found to be more effective than the TS scheme.

\section{CONCLUSION}

In this paper, we have proposed a novel sequential resource distribution (SRD) technique for cooperative multi-user multirelay OFDM networks with relay selection where the endusers are capable of performing joint data processing and energy harvesting. We addressed the problem of maximizing the sum-rate of end-users constrained to individual source and relays' transmit power and harvested energy at the users. Furthermore, this scenario was studied for both TS and PS based SWIPT receiver architectures. Using the numerical simulations, we demonstrated the effectiveness of the proposed SRD technique in contrast to a semi-random scheme and provided a comparative study between the TS and PS schemes. In future, we plan to extend this work by solving the dual optimization problem of $(P 1)$ and show the asymptotic optimality of this solution when the number of sub-carriers $N$ is large enough.

\section{ACKNOWLEDGMENT}

This work was partially supported by the Luxembourg National Research Fund (FNR) in the framework of the FNR-FNRS bilateral project "InWIP-NET : Integrated Wireless Information and Power Networks".

\section{REFERENCES}

[1] J. Yan, D. Wu, S. Sanyal, and R. Wang, "Trust-Oriented Partner Selection in D2D Cooperative Communications," IEEE Access, vol. 5, pp. 3444-3453, 2017.
[2] B. Nikfar and A. J. Han Vinck, "Relay selection in cooperative power line communication: A multi-armed bandit approach," Journal of Communications and Networks, vol. 19, no. 1, pp. 1-9, February 2017.

[3] T. Cover and A. E. Gamal, "Capacity theorems for the relay channel," IEEE Transactions on Information Theory, vol. 25, no. 5, pp. 572-584, September 1979.

[4] J. N. Laneman, D. N. C. Tse, and G. W. Wornell, "Cooperative diversity in wireless networks: Efficient protocols and outage behavior," IEEE Transactions on Information Theory, vol. 50, no. 12, pp. 3062-3080, December 2004.

[5] Y. Chen, R. Shi, W. Feng, and N. Ge, "AF Relaying With Energy Harvesting Source and Relay," IEEE Transactions on Vehicular Technology, vol. 66, no. 1, pp. 874-879, Jan 2017.

[6] X. Zhou, R. Zhang, and C.K. Ho, "Wireless Information and Power Transfer: Architecture Design and Rate-Energy Tradeoff," IEEE Trans. Commun., vol. 61, no. 11, pp. 4754-4767, Nov. 2013.

[7] T. D. P. Perera, D. N. K. Jayakody, S. K. Sharma, S. Chatzinotas, and J. Li, "Simultaneous Wireless Information and Power Transfer (SWIPT): Recent Advances and Future Challenges," IEEE Communications Surveys \& Tutorials, 2017.

[8] B. Yu, L. Liu, Y. Ma, P. Ma, and J. Tian, "Utility-based joint resource allocation for cooperative OFDM relaying in multiuser cognitive radio systems," in 2017 IEEE/CIC International Conference on Communications in China (ICCC), Oct 2017, pp. 1-6.

[9] W. Lu, Y. A. Zhang, M. Wang, X. Liu, and J. Hua, "Cooperative Spectrum Sharing in OFDM Two-Way Relay Systems With Bidirectional Transmissions," IEEE Communications Letters, vol. 21, no. 6, pp. 1349-1352, June 2017.

[10] A. El Shafie, K. Tourki, and N. Al-Dhahir, "An Artificial-NoiseAided Hybrid TS/PS Scheme for OFDM-Based SWIPT Systems," IEEE Communications Letters, vol. 21, no. 3, pp. 632-635, March 2017.

[11] S. Gautam and P. Ubaidulla, "Simultaneous transmission of information and RF energy in multicarrier systems," in 2016 23rd International Conference on Telecommunications (ICT). IEEE, 2016, pp. 1-5.

[12] Y. Lu, K. Xiong, Y. Zhang, P. Fan, and Z. Zhong, "Energy-Efficient Resource Allocation in OFDM Relay Networks under Proportional Rate Constraints," in 2016 IEEE Global Communications Conference (GLOBECOM), Dec 2016, pp. 1-6.

[13] S. Khakurel, L. Musavian, H. V. Vu, and T. Le-Ngoc, "Qos-aware utility-based resource allocation in mixed-traffic multi-user ofdm systems," IEEE Access, pp. 1-1, 2018

[14] Y. Li, W. Wang, J. Kong, and M. Peng, "Subcarrier Pairing for Amplifyand-Forward and Decode-and-Forward OFDM Relay Links," IEEE Commun. Lett., vol. 13, no. 4, pp. 209-211, Apr. 2009.

[15] I. Hammerstrom and A. Wittneben, "On the Optimal Power Allocation for Nonregenerative OFDM Relay Links," IEEE Int. Conf. on Communications, Istanbul, Turkey, 2006.

[16] Y. Liu, "Wireless Information and Power Transfer for MultirelayAssisted Cooperative Communication," IEEE Communications Letters, vol. 20, no. 4, pp. 784-787, April 2016.

[17] L. Liu, R. Zhang, and K. C. Chua, "Wireless Information Transfer with Opportunistic Energy Harvesting," IEEE Transactions on Wireless Communications, vol. 12, no. 1, pp. 288-300, January 2013.

[18] K. Xiong, P. Fan, C. Zhang, and K. Letaief, "Wireless Information and Energy Transfer for Two-Hop Non-Regenerative MIMO-OFDM Relay Networks," IEEE J. Sel. Area Commun., vol. 33, no. 8, pp. 1595-1611, Aug. 2015.

[19] Y. Liu and X. Wang, "Information and Energy Cooperation in OFDM Relaying: Protocols and Optimization," IEEE Trans. Veh. Technol., vol. 65, no. 7, pp. 5088-5098, Jul. 2016.

[20] S. Gautam, E. Lagunas, S.K. Sharma, S. Chatzinotas, and B. Ottersten, "Relay Selection Strategies for SWIPT-Enabled Cooperative Wireless Systems," IEEE Int. Symp. Personal, Indoor and Mobile Radio Communications (PIMRC), Montreal, Canada, Oct. 2017.

[21] S. Gautam and P. Ubaidulla, "Relay Selection and Transceiver Design for Joint Wireless Information and Energy Transfer in Cooperative Networks," in 85th Vehicular Technology Conference (VTC), 2017Spring, June 2017.

[22] L. Liu, R. Zhang, and K.C. Chua, "Wireless Information and Power Transfer: A Dynamic Power Splitting Approach," IEEE Trans. Commun., vol. 61, no. 9, pp. 3990-4001, Sep. 2013.

[23] W. Dang, M. Tao, H. Mu, and J. Huang, "Subcarrier-Pair Based Resource Allocation for Cooperative Multi-Relay OFDM Systems," IEEE Trans. Wireless Commun., vol. 9, no. 5, pp. 1640-1649, May 2010. 